\title{
Spreading of multiple Listeria monocytogenes abscesses via central nervous system fiber tracts: case report
}

\author{
Michel W. Bojanowski, MD, FRCSC, ${ }^{1}$ Romuald Seizeur, MD, PhD, ${ }^{1,3}$ Khaled Effendi, MD, FRCSC, ${ }^{1}$ \\ Patrick Bourgouin, MD, ${ }^{1}$ Elsa Magro, MD, ${ }^{1}$ and Laurent Letourneau-Guillon, MD, FRCPC ${ }^{2}$
}

\begin{abstract}
Divisions of ${ }^{1}$ Neurosurgery and ${ }^{2}$ Neuroradiology, Hôpital Notre Dame, Montréal, Quebec, Canada; and ${ }^{3}$ Service de Neurochirurgie, CHRU de Brest, France
\end{abstract}

\begin{abstract}
Animal studies have shown that Listeria monocytogenes can probably access the brain through a peripheral intraneural route, and it has been suggested that a similar process may occur in humans. However, thus far, its spreading through the central nervous system (CNS) has not been completely elucidated. The authors present a case of multiple L. monocytogenes cerebral abscesses characterized by a pattern of distribution that suggested spread along white matter fiber tracts and reviewed the literature to identify other cases for analysis. They elected to include only those cases with 3 or more cerebral abscesses to make sure that the distribution was not random, but rather followed a pattern. In addition, they included those cases with abscesses in both the brainstem and the cerebral hemispheres, but excluded cases in which abscesses were located solely in the brainstem.
\end{abstract}

Of 77 cases of $L$. monocytogenes CNS abscesses found in the literature, 17 involved multiple abscesses. Of those, 6 were excluded for lack of imaging and 3 because they involved only the brainstem. Of the 8 remaining cases from the literature, one was a case of bilateral abscesses that did not follow a fiber tract; another was also bilateral, but with lesions appearing to follow fiber tracts on one side; and in the remaining 6 , to which the authors added their own case for a total of 7 , all the abscesses were located exclusively in the same hemisphere and distributed along white matter fiber tracts.

The findings suggest that after entering the CNS, L. monocytogenes travels within the axons, resulting in a characteristic pattern of distribution of multiple abscesses along the white matter fiber tracts in the brain. This report is the first description suggesting intraaxonal CNS spread of $L$. monocytogenes infection in humans following its entry into the brain. This distinct pattern is clearly seen on imaging and its recognition may be valuable in the diagnosis of listeriosis. This finding may allow for earlier diagnosis, which may improve outcome.

http://thejns.org/doi/abs/10.3171/2014.12.JNS142100

KEY WORDS Listeria monocytogenes; brain abscesses; fiber tracts; bacterial neural invasion; diagnosis; neuroradiology; infection

$L$ ISTERIA monocytogenes is a facultative intracellular pathogen that may infect the central nervous system (CNS). ${ }^{15,30}$ When the CNS is affected by L. monocytogenes, most of the time it causes meningitis; however, the organism is well known for its classic involvement of the rhombencephalon. ${ }^{3,40}$ Rarely, it may be responsible for brain abscesses. ${ }^{24,31}$

L. monocytogenes can enter the CNS via hematogenous dissemination, but studies suggest that it can also reach the CNS by traveling along the axons of nerves in the peripheral nervous system, such as the trigeminal cranial nerve, which would explain the associated characteristic rhombencephalitis. ${ }^{3,41}$
Within the CNS, it has not yet been established whether the intraaxonal route along the fiber tracts may also account for spreading of L. monocytogenes throughout the brain. ${ }^{33}$ Such spreading has been suggested in animal studies. ${ }^{7}$ One way to investigate this possibility in humans is to analyze the pattern of distribution of multiple abscesses on imaging studies.

In this paper we report on a patient with multiple $L$. monocytogenes abscesses in whom we observed a pattern of distribution along white matter fiber tracts. We also present the results of a literature search for cases of cerebral L. monocytogenes infection involving 3 or more CNS abscesses, as well as our analysis of the pattern of distribu-

ABBREVIATIONS CNS = central nervous system; DTI = diffusion tensor imaging; SWI = susceptibility-weighted imaging

SUBMITTED September 11, 2014. ACCEPTED December 4, 2014.

INCLUDE WHEN CITING Published online June 19, 2015; DOI: 10.3171/2014.12.JNS142100.

DISCLOSURE The authors report no conflict of interest concerning the materials or methods used in this study or the findings specified in this paper. 
tion in these cases and our own. We hypothesize that if $L$. monocytogenes also uses the intraneural route to spread through the CNS, multiple brain abscesses would follow white matter tracts and thus would be seen on imaging studies in a distinct pattern of distribution and readily recognized as listeriosis.

\section{Case Report}

A 74-year-old woman who was known to have high blood pressure, Type 2 diabetes, and obesity presented with a brief history of neurological symptoms. She had had a progressive headache for 1 week, and then her family had noticed confusion and psychomotor slowing. A few days later she had begun to demonstrate progressive left hemiparesis involving both limbs as well as the face. Upon neurological examination, she was found to be drowsy but oriented. Assessment of motor function revealed a $4 / 5$ left paresis, but the results of sensory examination were normal. Assessment of her cranial nerves revealed a mild left facial central paresis. Examination of the other cranial nerves showed no abnormality. She was afebrile.

Initial laboratory studies showed an elevated white blood cell count, hyponatremia $(126 \mathrm{mmol} / \mathrm{L}$ [reference range $135-145 \mathrm{mmol} / \mathrm{L}])$, and a decreased level of urea $(1.7 \mathrm{mmol} / \mathrm{L}$ [reference range $3.3-8.8 \mathrm{mmol} / \mathrm{L}$ ], with the creatine level at $46 \mu \mathrm{mol} / \mathrm{L}$ [reference range $42-89$ $\mu \mathrm{mol} / \mathrm{L}])$. The hyponatremia was thought to be secondary to a syndrome of inappropriate antidiuretic hormone secretion caused by hydrochlorothiazide therapy and was treated accordingly.

Initial MRI revealed a cluster of rounded and ovoid lesions within the subcortical white matter of the right frontal lobe, deep to the precentral gyrus and anteriorly within the posterior superior frontal gyrus. These lesions all shared common imaging features, including a rim of spontaneous T1 hyperintensity; concentric iso- to hypointense rings and a hypointense core on T2 and blooming artifacts on susceptibility-weighted imaging (SWI); and they showed rim enhancement as well as perifocal vasogenic edema. A few lesions were located in the juxta-cortical white matter and seemed to follow the U-fibers (Fig. 1). An infectious cause was deemed possible, and complete infectious screening was performed, including transesophageal ul- trasonography, and treatment was initiated with vancomycin and ceftriaxone. The patient's condition deteriorated 3 days after admission, with increase facial asymmetry and $3 / 5$ left paresis. Additional imaging showed an increased number of lesions.

Culture of blood specimens obtained on admission was positive for gram-positive bacilli. L. monocytogenes was suspected, and ampicillin was added to the intravenous antibiotic treatment.

On follow-up imaging studies performed 12 days later, improvement in the size and enhancement of the initial cluster was noted, but new lesions had appeared more anteriorly in the frontal lobe, deep to the superior and middle frontal gyri (Fig. 2). The latter group of lesions apparently followed the superior longitudinal fasciculus and short association fibers. Other lesions had also developed deeper within the corona radiata, the posterior limb of the internal capsule, and the cerebral peduncle, all following along the corticospinal tract (Figs. 3 and 4). The pattern of spread of these lesions fitted the distribution of the major white matter tracts as seen on diffusion tensor imaging (DTI) (Figs. 1, 3, and 4). All lesions subsequently began to resolve with intravenous antibiotic therapy, and no new lesions were seen on further imaging performed up to 6 weeks after the initial presentation.

The patient's condition improved progressively, and she demonstrated complete recovery of muscle strength and awareness after adjustment of her antibiotic therapy, when ampicillin was added to the initial regimen of vancomycin and ceftriaxone. She left the hospital for a rehabilitation center on intravenous antibiotics.

\section{Literature Review}

We reviewed the literature searching for cerebral $L$. monocytogenes cases involving 3 or more CNS abscesses and retained those where sufficient imagery was available, adding our own case. The images were analyzed to determine whether the pattern of distribution was along the white matter fiber tracts. We elected to include only those cases with 3 or more abscesses in order to make sure that the distribution was not random, but rather followed a pattern. In addition, we included those cases with abscesses
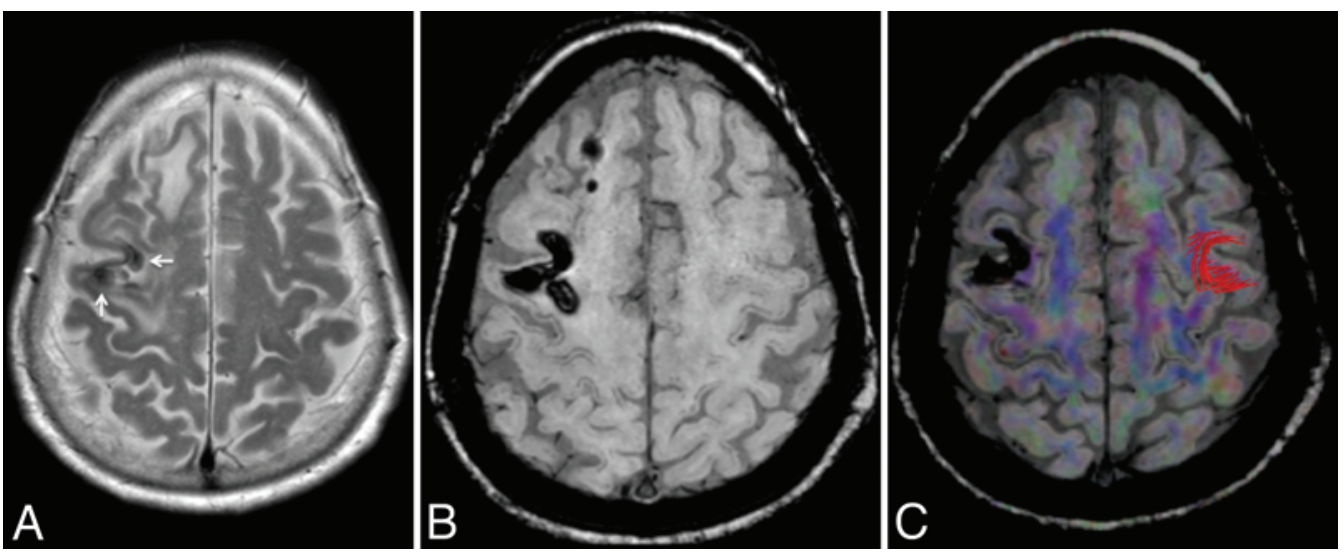

FIG. 1. Right frontal lesions on axial T2-weighted image (A), axial susceptibility-weighted image (B), and fusion of SWI and combined fractional anisotropy/directional map (C). At least 2 lesions seem to follow the short association fibers (U-fibers [arrows in A]). 

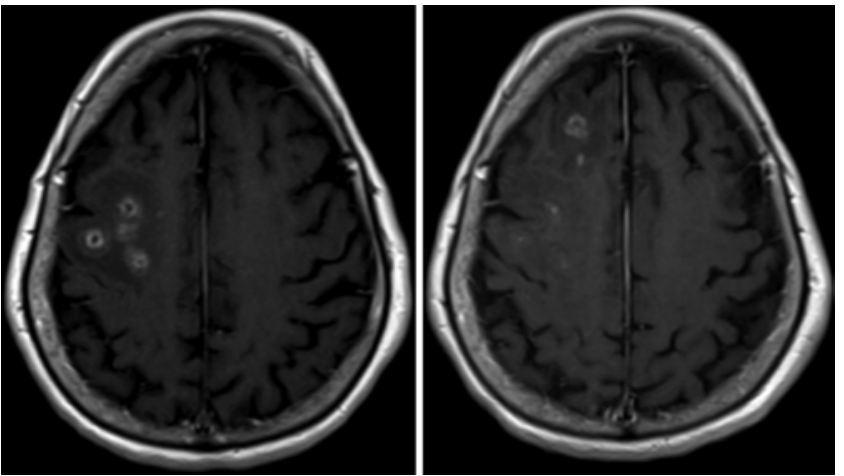

FIG. 2. Axial T1-weighted MR images obtained on admission (left) and Day 12 (right) showing the initial cluster of lesions within the subcortical white matter of the precentral gyrus as well as the superior and middle frontal gyri with subsequent anterior spread on follow-up imaging.

in both the brainstem and at least 1 cerebral hemisphere, but excluded those in which abscesses were located solely in the brainstem.

Beginning with Limmahakhun and Chayakulkeeree's review of L. monocytogenes brain abscesses published until $2011,{ }^{23}$ to which we added 11 other cases published during the same study period, we reviewed ${ }^{1,5,9,18,22,34,35}$ the literature from 2011 to 2014 and added 9 additional cases. ${ }^{6,8,11,16,19,26,36,39}$ We found a total of 77 cases of L. monocytogenes brain abscesses, of which 17 involved multiple abscesses. Of those, 6 were excluded for lack of imagery. $4,10,20,21$ Of the remaining 11,3 involved the brainstem without involving the cerebral hemispheres and thus were excluded. ${ }^{6,25,28}$ In the 8 final cases from the literature, 1 case was of bilateral abscesses that did not follow a fiber tract, ${ }^{23} 1$ case was also bilateral but with abscesses (possibly) following the fiber tracts on 1 side, ${ }^{38}$ while in the remaining $6,1,16,26,29,37$ to which we added our own case for a total of 7, all the abscesses were located exclusively in the same hemisphere and were distributed along white matter fiber tracts (Table 1). In the majority of cases, the distribution was along the projection fiber tracts toward the brainstem, but in some cases, as in ours, the short and long association fibers were also affected. In our own case, where we had access to all views of the images, even though multiple tracts were involved, there was a main cluster of lesions in 1 site where all the tracts intersected.

\section{Discussion}

Listeriosis is a bacterial infection most commonly caused, in humans, by L. monocytogenes and primarily affects the CNS-often in immune-compromised patients. ${ }^{5,30}$ but it can also be seen in immune-competent patients. The bacterium is acquired via the oral route, and the ensuing infection is usually transmitted through septicemia, most commonly developing into meningitis. ${ }^{24}$ At times, it can also manifest as encephalitis with a characteristic predilection for the brainstem. Rarely, it develops into brain abscesses, and in those cases the outcome is often grim. ${ }^{14,30}$

Animal studies have shown that L. monocytogenes can also enter the CNS by traveling within the axons of the trigeminal nerve. In these studies, inoculation of L. monocytogenes into lips, oral mucosa, or dental pulp resulted in encephalitis confined to the brainstem. Neuro-pathological lesions were observed in the trigeminal nerve and ganglion on the side of the injection. ${ }^{13}$ In other studies, electron microscopy revealed the presence of L. monocytogenes within the axons of the trigeminal nerve.,33

Since listeriosis is mainly contracted through ingestion of contaminated food, it is then not surprising that the trigeminal nerve, which is connected to the pons, carries the bacteria, resulting in the characteristic, albeit not frequent in humans, rhombencephalitis.

Other clinical and experimental studies have also suggested that the trigeminal nerve is not the only nerve to carry the bacteria and that other cranial or peripheral nerves may also be pathways for invasion of the CNS., ${ }^{2,40}$
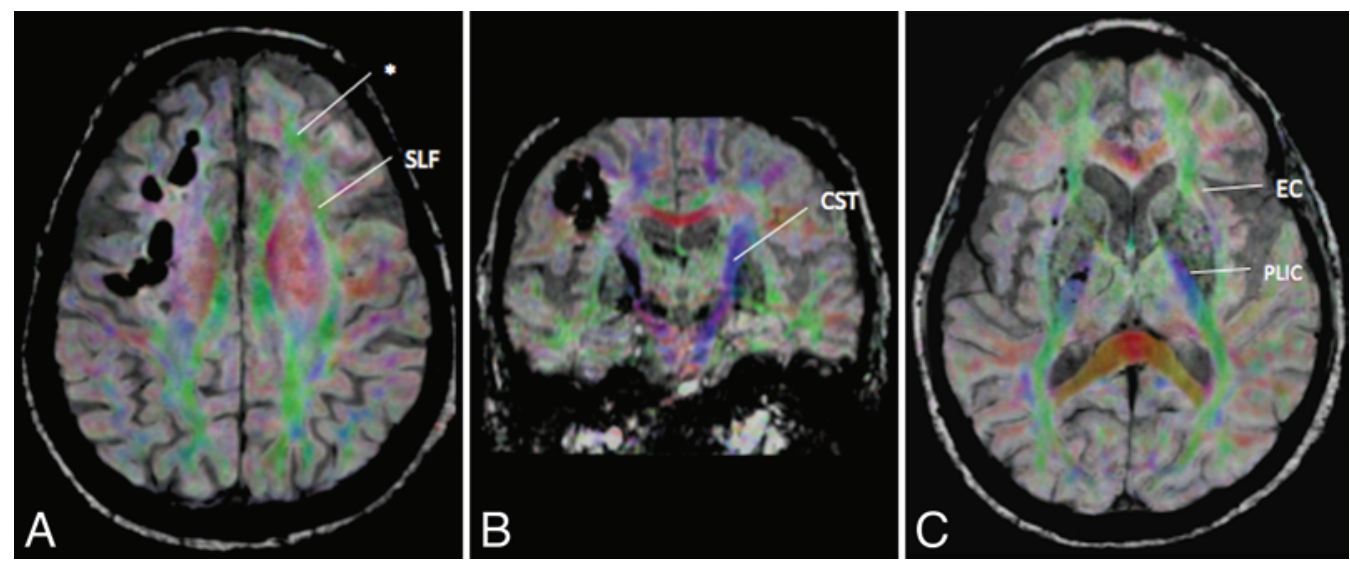

FIG. 3. Fused images generated from SWI and combined fractional anisotropy/directional maps (DTI) showing the spread from the subcortical white matter of the right precentral gyrus to the anterior right frontal lobe involving the superior longitudinal fasciculus (SLF) and short-range association fibers (indicated by asterisk) (A), the deep white matter along the corticospinal tract (CST) (B) and the external capsule (EC) on the same side (along fibers of the SFL, uncinate fasciculus, and inferior fronto-occipital fasciculus) (C). Also note the presence of lesions at the level of the posterior limb of the internal capsule (PLIC) in panel C. All labels are contralateral to the lesions (the latter disrupted proper visualization of these major tracts). 

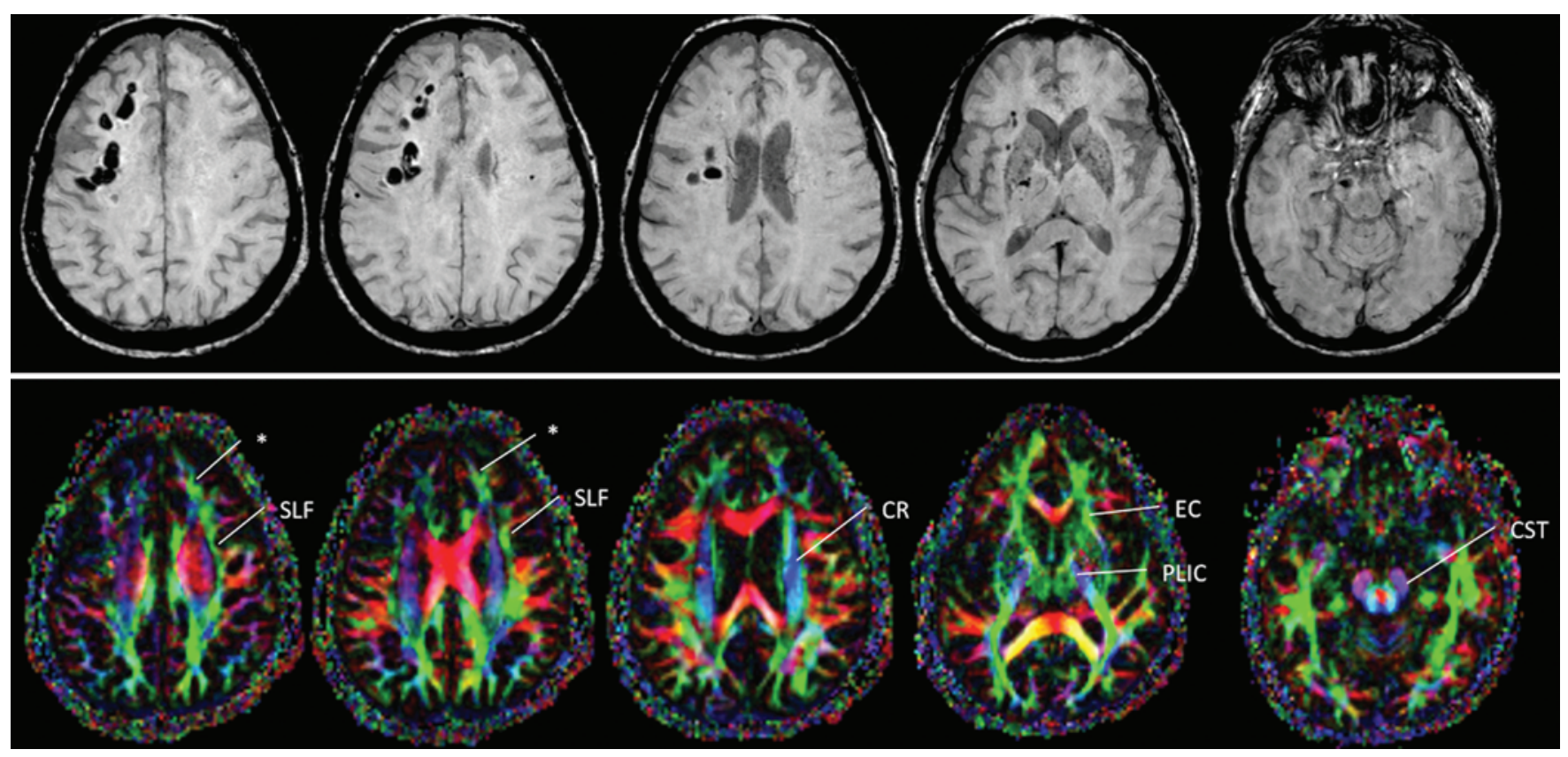

FIG. 4. SWI (upper) and combined fractional anisotropy/directional maps obtained from DTI (lower) show that the lesions were located in the subcortical white matter of the right precentral gyrus and subsequently spread to the anterior right frontal lobe involving the superior longitudinal fasciculus (SLF) and short association fibers (indicated by asterisk). The lesions also appear to follow the corticospinal tract (CST), including the corona radiata (CR), posterior limb of the internal capsule (PLIC), and cerebral peduncle. Some lesions were also visible within the external capsule on the same side. Incidental subcortical microhemorrhages were also evident within both hemispheres on SWI. EC indicates external capsule, including fibers of the SLF, uncinate fasciculus, and inferior fronto-occipital fasciculus.

It has been found that the bacterium enters the cell by endocytosis and multiplies within the cytosol, where it acquires an actin tail that propels it through the axon as well as to neighboring cells. ${ }^{12}$ L. monocytogenes is able to survive and multiply in a wide variety of cell types, including macrophages and neurons. ${ }^{13,17,32}$

Even though studies have suggested that L. monocytogenes can use a peripheral intraneural route to invade the CNS, it has not yet been established how it then spreads throughout the brain. In fact, in one study, peripheral neurons were able to be effectively infected, contrary to hippocampal neurons, which were resistant, suggesting that different types of neurons might have different susceptibilities to bacterial tropism.13,17 A recent animal study, however, suggests that once the bacteria have gained access to the CNS via the peripheral nervous system, the infection can spread along the axons, producing additional lesions. ${ }^{17,32}$ This hypothesis has not previously been evaluated in humans. Our results based on MRI in cases of multiple brain abscesses strongly suggest that this intraneural mechanism of spreading in the CNS also applies to humans. This is the first report, to our knowledge, that demonstrates central intraneural spreading of CNS listeriosis in humans.

In our study, we excluded patients with abscesses limited to the brainstem because these abscesses can cover a large area that includes many overlapping fiber tracts, making it difficult to assess the involvement of specific tracts. In 8 of the 9 cases of multiple abscesses analyzed in this study, the abscesses followed a fiber tract. In addition, the fact that in 7 of those cases, all the abscesses were limited to a single hemisphere, does not favor hematogenous invasion and adds to the evidence that once within the CNS, L. monocytogenes travels intraaxonally. Based on the locations of the multiple abscesses, which are visible on imaging studies in the literature, for each case, we have provided a drawing linking the abscesses, thus obtaining a trajectory that reflects the pathway of white fiber tracts (Table 1). We acknowledge that these drawings represent extrapolation, given that the literature provided limited images and only general description, since preceding authors had not made the link between the location of abscesses and the pathway of white matter tracts. This is the first such observation in humans.

The frequency of axonal spreading in cases of Listeria infection of the central nervous system is not known. Since the link between the location of abscesses and white fiber tracts has not been made before, retrospective review cannot provide this information. Also, since sufficient imagery is needed to make such an observation, for this reason also, only 6 of 77 cases were suitable for our analysis. We cannot determine the frequency or the incidence of spreading via white fiber tracts, but the goal of our paper is to demonstrate that such spreading is possible and when present highly suggests the presence of Listeria. This form of spreading can not be seen on imaging studies in cases of meningitis and rhombencephalitis, but that does not exclude such a possibility even in these cases.

In our case, because of the location of the main concentration of the abscesses, the initial invasion was probably 
TABLE 1. Fiber tracts involved in patients with multiple L. monocytogenes abscesses*

\begin{tabular}{|c|c|c|c|c|}
\hline Authors \& Year & Patient Age (yrs) & Main Tracts Involved & Schematic Representation & Clinical Evolution \\
\hline Gertz et al., 2013 & 70 & Projection fibers & & $\begin{array}{l}\text { Minor neurological } \\
\text { sequelae }\end{array}$ \\
\hline Matera et al., 2012 & 57 & $\begin{array}{l}\text { Projection fibers, long associa- } \\
\text { tion fibers }\end{array}$ & & Dead \\
\hline Mukherjee et al., 2011 & 55 & Projection fibers & & Good \\
\hline $\begin{array}{l}\text { Soares-Fernandes et al., } \\
2008\end{array}$ & 46 & Projection fibers & & $\begin{array}{l}\text { Minor neurological } \\
\text { sequelae }\end{array}$ \\
\hline Aladro et al., 1996 & 38 & Projection fibers & & $\begin{array}{l}\text { Minor neurological } \\
\text { sequelae }\end{array}$ \\
\hline
\end{tabular}


TABLE 1. Fiber tracts involved in patients with multiple L. monocytogenes abscesses* (continued)

\begin{tabular}{|c|c|c|c|c|}
\hline Authors \& Year & Patient Age (yrs) & Main Tracts Involved & Schematic Representation & Clinical Evolution \\
\hline Aladro et al., 1996 & 65 & Projection fibers & & $\begin{array}{l}\text { Minor neurological } \\
\text { sequelae }\end{array}$ \\
\hline Present case & 74 & $\begin{array}{l}\text { Projection fibers, long associa- } \\
\text { tion fibers, short association } \\
\text { fibers (U fibers) }\end{array}$ & & Good \\
\hline
\end{tabular}

* Data from patients with L. monocytogenes abscesses following fiber tracts.

via the hematogenous route. Blood culture confirmed the presence of L. monocytogenes in our case, as in $86 \%$ of cases in the literature. ${ }^{14}$ We hypothesize that subsequent spreading proceeded intraaonally. Such invasion along the nerve fibers would explain why there is much more neurological deficit in patients with L. monocytogenes-related abscesses ${ }^{14,27}$ than in patients with other types of brain abscesses in which the infection expands only locally.

This particular form of spreading would produce a typical pattern of distribution of the abscesses, based on the organization of the fiber tracts of the CNS. Since no other bacteria are known to travel in this way, this distinct radiological presentation may have significant diagnostic value, particularly when the results of blood studies are negative and lumbar puncture is contraindicated. Given the often grim prognosis of L. monocytogenes abscesses, recognizing the characteristic pattern of distribution may lead to earlier diagnosis, allowing for quicker antibiotic treatment and thus leading to better outcome.

\section{Conclusions}

Our findings suggest that once it has entered in the CNS, L. monocytogenes travels within the axons, resulting in a characteristic pattern of distribution of multiple abscesses along the white matter fiber tracts in the brain. This distinct pattern is clearly seen on imaging studies and its recognition may be valuable in the diagnosis of $L$. monocytogenes infection. This finding may allow for earlier diagnosis, which may improve outcome. This report is the first description suggesting intraaxonal CNS spreading of L. monocytogenes infection in humans.

\section{Acknowledgments}

We would like to thank Ms. Gislaine Plante for the illustrations in the table and Mrs. Lucyna Szpak for her assistance in editing the manuscript.

\section{References}

1. Aladro Y, Ponce P, Santullano V, Angel-Moreno A, Santana MA: Cerebritis due to Listeria monocytogenes: CT and MR findings. Eur Radiol 6:188-191, 1996

2. Antal EA, Løberg EM, Bracht P, Melby KK, Maehlen J: Evidence for intraaxonal spread of Listeria monocytogenes from the periphery to the central nervous system. Brain Pathol 11:432-438, 2001

3. Armstrong RW, Fung PC: Brainstem encephalitis (rhombencephalitis) due to Listeria monocytogenes: case report and review. Clin Infect Dis 16:689-702, 1993

4. Banerji A, Noya FJ: Brain abscess associated with neonatal listeriosis. Pediatr Infect Dis J 18:305-307, 1999

5. Berenguer J, Solera J, Diaz MD, Moreno S, López-Herce JA, Bouza E: Listeriosis in patients infected with human immunodeficiency virus. Rev Infect Dis 13:115-119, 1991

6. Beynon C, Neumann JO, Bösel J, Unterberg AW, Kiening KL: Stereotactic biopsy and drainage of a brainstem abscess caused by Listeria monocytogenes. Neurol Med Chir (Tokyo) 53:263-265, 2013

7. Charlton KM: Spontaneous listeric encephalitis in sheep. Electron microscopic studies. Vet Pathol 14:429-434, 1977

8. Choudhury N, Khan AB, Tzvetanov I, Garcia-Roca R, Oberholzer J, Benedetti E, et al: Cerebellar abscess caused by Listeria monocytogenes in a liver transplant patient. Transpl Infect Dis 15:E224-E228, 2013

9. Chow AW, Alexander E, Montgomerie JZ, Guze LB: Successful treatment of non-meningitic listerial brain abscess without operation. West J Med 122:167-171, 1975 
10. Cone LA, Leung MM, Byrd RG, Annunziata GM, Lam RY, Herman BK: Multiple cerebral abscesses because of Listeria monocytogenes: three case reports and a literature review of supratentorial listerial brain abscess(es). Surg Neurol 59:320-328, 2003

11. Coste JF, Duval V, Nguyen Y, Guillard T, Brasme L, David $\mathrm{C}$, et al: [Unusual location of a brain abscess due to Listeria monocy togenes.] Pathol Biol (Paris) 60:e45-e48, 2012 (Fr)

12. Dons L, Jin Y, Kristensson K, Rottenberg ME: Axonal transport of Listeria monocytogenes and nerve-cell-induced bacterial killing. J Neurosci Res 85:2529-2537, 2007

13. Dons L, Weclewicz K, Jin Y, Bindseil E, Olsen JE, Kristensson K: Rat dorsal root ganglia neurons as a model for Listeria monocytogenes infections in culture. Med Microbiol Immunol (Berl) 188:15-21, 1999

14. Eckburg PB, Montoya JG, Vosti KL: Brain abscess due to Listeria monocytogenes: five cases and a review of the literature. Medicine (Baltimore) 80:223-235, 2001

15. Gellin BG, Broome CV: Listeriosis. JAMA 261:1313-1320, 1989

16. Gertz K, Siebert E, Halle E, Kronenberg G, Endres M, Klingebiel R, et al: Multiple supratentorial brain abscesses due to Listeria monocytogenes in a patient with myasthenia gravis. Clin Neurol Neurosurg 115:1923-1924, 2013

17. Guldimann C, Lejeune B, Hofer S, Leib SL, Frey J, Zurbriggen A, et al: Ruminant organotypic brain-slice cultures as a model for the investigation of CNS listeriosis. Int J Exp Pathol 93:259-268, 2012 Available

18. Haykal H, Zamani A, Wang AM, Barsotti J: CT features of early Listeria monocytogenes cerebritis. AJNR Am J Neuroradiol 8:279-282, 1987

19. Horta-Baas G, Guerrero-Soto O, Barile-Fabris L: Central nervous system infection by Listeria monocy togenes in patients with systemic lupus erythematosus: analysis of 26 cases, including the report of a new case. Reumatol Clin 9:340-347, 2013

20. Kennard C, Howard AJ, Scholtz C, Swash M: Infection of the brainstem by Listeria monocytogenes. J Neurol Neurosurg Psychiatry 42:931-933, 1979

21. Larsson S, Linell F: Correlations between clinical and postmortem findings in listeriosis. Scand J Infect Dis 11:55-58, 1979

22. Leung R, Woo E, Yu YL, Huang CY: Listeria brain abscess associated with steroid therapy: successful non-surgical treatment. Clin Exp Neurol 24:181-186, 1987

23. Limmahakhun S, Chayakulkeeree M: Listeria monocytogenes brain abscess: two cases and review of the literature. Southeast Asian J Trop Med Public Health 44:468-478, 2013

24. Lorber B: Listeriosis. Clin Infect Dis 24:1-11, 1997

25. Maezawa Y, Hirasawa A, Abe T, Kawamura H, Sekiguchi K, Kunimoto M, et al: Successful treatment of listerial brain abscess: a case report and literature review. Intern Med 41:1073-1078, 2002

26. Matera G, Puccio R, Giancotti A, Quirino A, Guadagnino $\mathrm{V}$, Pardatscher K, et al: Multiple abscesses of the left brain hemisphere due to Listeria monocytogenes in an immunocompromised patient: a case report. Infez Med 20:279-283, 2012

27. Mathisen GE, Johnson JP: Brain abscess. Clin Infect Dis 25:763-781, 1997

28. Mrowka M, Graf LP, Odin P: MRI findings in mesenrhombencephalitis due to Listeria monocytogenes. J Neurol Neurosurg Psychiatry 73:775, 2002
29. Mukherjee P, Mazumdar S, Ahmed A, Chakraborty S, Bhowmik J, Jana S, et al: Unusual presentation of brain abscess with uncommon organism in an immunocompetent person. J Assoc Physicians India 59:453-455, 2011

30. Mylonakis E, Hohmann EL, Calderwood SB: Central nervous system infection with Listeria monocytogenes. 33 years' experience at a general hospital and review of 776 episodes from the literature. Medicine (Baltimore) 77:313-336, 1998

31. Nieman RE, Lorber B, Reviews S, Apr NM: Listeriosis in adults: a changing pattern. Report of eight cases and review of the literature, 1968-1978. Rev Infect Dis 2:207-227, 1980

32. Oevermann A, Di Palma S, Doherr MG, Abril C, Zurbriggen A, Vandevelde M: Neuropathogenesis of naturally occurring encephalitis caused by Listeria monocytogenes in ruminants. Brain Pathol 20:378-390, 2010

33. Otter A, Blakemore WF: Observation on the presence of Listeria monocytogenes in axons. Acta Microbiol Hung 36:125-131, 1989

34. Ricard D, Sallansonnet-Froment M, Defuentes G, de Greslan T, Bounolleau P, Taillia H, et al: [Listeria monocytogenes abscess of the brain.] Rev Neurol (Paris) 164:388-393, 2008 (Fr)

35. Samra Y, Hertz M, Altmann G: Adult listeriosis-a review of 18 cases. Postgrad Med J 60:267-269, 1984

36. Sedney CL, Harshbarger T, Gyure K: An unusual form of listerial CNS infection. W V Med J 108:12-15, 2012

37. Soares-Fernandes JP, Beleza P, Cerqueira JJ, Ribeiro M, Maré R, Lourenço E, et al: Simultaneous supratentorial and brainstem abscesses due to Listeria monocytogenes. J Neuroradiol 35:173-176, 2008

38. Stefanovic A, Reid J, Nadon AC, Grant J: Potential nosocomial acquisition of epidemic Listeria monocytogenes presenting as multiple brain abscesses resembling nocardiosis. Can J Infect Dis Med Microbiol 21:57-60, 2010

39. Tseng J, Strasfeld LM, Orloff SL: An unusual presentation of altered mental status after orthotopic liver transplantation: Listeria brain abscess. Transplantation 95:e72-e73, 2013

40. Uldry PA, Kuntzer T, Bogousslavsky J, Regli F, Miklossy $\mathrm{J}$, Bille J, et al: Early symptoms and outcome of Listeria monocytogenes rhombencephalitis: 14 adult cases. J Neurol 240:235-242, 1993

41. Vázquez-Boland JA, Kuhn M, Berche P, Chakraborty T, Domínguez-Bernal G, Goebel W, et al: Listeria pathogenesis and molecular virulence determinants. Clin Microbiol Rev 14:584-640, 2001

\section{Author Contributions}

Conception and design: Bojanowski. Acquisition of data: Bojanowski, Seizeur, Effendi, Bourgouin, Letourneau-Guillon. Analysis and interpretation of data: Bojanowski, Seizeur, Magro, Letourneau-Guillon. Drafting the article: Bojanowski. Critically revising the article: Bojanowski, Seizeur, Magro, LetourneauGuillon. Reviewed submitted version of manuscript: all authors. Approved the final version of the manuscript on behalf of all authors: Bojanowski. Administrative/technical/material support: Bojanowski. Study supervision: Bojanowski, Seizeur.

\section{Correspondence}

Michel W. Bojanowski, Division of Neurosurgery, Hôpital Notre Dame, 1560 Sherbrooke Est, Montréal, QC H2L 4M1, Canada. email: michel.bojanowski.chum@ssss.gouv.qc.ca. 